\title{
Value-Instantiating Beliefs: A Practical Substitute for Meaning
}

\author{
Vladimir Ponizovskiy \\ Jacobs University, Bremen, Germany
}

\begin{abstract}
The construct of "meaning", although commonly used by psychologists, does not have a formal operationalization. In this article I specify a construct of value-instantiating belief, intended to quantify individual differences in motivationally relevant aspects of meaning. A value-instantiating belief is a personal belief about consequences of an object, action, or event for a personal value. I describe predictors and consequences of value-instantiating beliefs, their role in attitude formation, and potential applications in research on values, intergroup relations, persuasion, and culture. Using value-instantiating beliefs to assess individual interpretations of psychological stimuli can aid in specifying and testing boundary conditions for psychological effects and improving the predictive power of psychological models. The proposed construct can further generate new research directions that address questions about meaning.
\end{abstract}

Keywords: meaning, motivation, values, attitudes

Psychology is faced with a "replicability crisis"- the observation that as often as not, psychological studies fail to produce similar results when replicated (Open Science Collaboration, 2015). This undermines confidence in the outcomes of psychological research.

Psychological studies may fail to replicate for two types of reasons. First, studies may not replicate when the original study produced a false positive result: it found an effect where there was none. This problem can be addressed by improving the standards of study design to reduce the probability of false positives occurring. Much of the response to the "replicability crisis" promoted solutions of these type: ensuring the studies are properly powered (Fraley \& Vazire, 2014), combatting questionable research practices such as selecting the results from a study that "look good" (John et al., 2012), and reducing publication bias (Francis, 2012).

Alternatively, psychological research may fail to replicate because of the context-dependency of the original study (Swiatkowski \& Dompnier, 2017): the effect was there, but it was conditional on specific circumstances that were not reproduced in the replication study ${ }^{1}$ Context-dependency of psychological studies contributes to the failure to replicate over and above methodological characteristics, such as statistical power and size of the effect (Van Bavel et al., 2016a), and may affect social psychology more than other subdisciplines of psychology (Inbar, 2016, Van Bavel et al., 2016b). Van Bavel and colleagues aptly cite John Touhey, criticizing the "dubious ... assumption that experimental manipulations can be studied

Correspondence should be addressed to Vladimir Ponizovskiy, 309 South Hall, Campus Ring 1, Bremen, Germany, 28759. Email: vladimir.ponizovskiy@gmail.com apart from the cultural and historical contexts that define their meanings" (Touhey, 1981, p. 594). While psychologists generally acknowledge that historical, cultural, and social contexts give meaning to the manipulations, dependent and explanatory variables in psychological research (Jost \& Kruglanski, 2002), there are few theoretical constructs that could help formalize what these meanings are, how they arise from the context, how they influence psychological mechanisms, and whether they differ or stay the same between two specific cultures, times, or settings.

Recommendations for addressing context sensitivity are often vague or difficult to implement, especially compared to straightforward methodological recommendations for avoiding false positives. Van Bavel and colleagues recommend for psychologists "to be as explicit as possible in defining likely contextual boundaries on individual effects, and to assess those boundaries across multiple studies" (Van Bavel et al., 2016a, p. 6457). Yet, in their own study, the authors did not theorize about specific contextual boundaries to studied effects, and did not try to assess them objectively, instead relying on expert ratings. In a recent discussion of the "generalizability crisis", Tal Yarkoni (Yarkoni, 2019) proposes to not make a priori predictions as to which contextual factors will be relevant to a psychological effect and instead model all potentially contributing factors as random effects - an approach hardly feasible given that the list of such factors is limitless.

In this paper, I specify the construct of a value in-

\footnotetext{
${ }^{1}$ The two threats to replicability are not independent: among psychologists, the increased costs borne by collecting larger samples seem to be compensated by using inexpensive study designs (Sassenberg \& Ditrich, 2019). Reliance on such designs further reduces the diversity and representativeness of samples, undermining generalizability (Henrich et al., 2010).
} 
stantiating belief (VIB) as a quantifiable operationalization of contextual "meaning". This construct allows developing theoretical arguments about contextual boundaries of psychological effects, quantitatively evaluating such boundary conditions, and improving the predictive power of psychological models.

\section{Meaning as a Boundary Condition in Psychological Research}

It is a truism that psychological processes are shaped not by the objective characteristics of the environment, but rather by the subjective meaning that people extract from it: subjective income is more important for life satisfaction than objective income (Ackerman \& Paolucci, 1983), perceived threat can lead to prejudice even if there are no objective grounds to it (Stephan \& Stephan, 2000), perceived rather than actual similarity relates to interpersonal attraction in close relationships (Montoya et al., 2008). Subjective interpretations of reality differ between situations, times, and individuals: the amount of 2019 US dollars that constitutes a satisfactory income depends on the country, the year, and the person making the judgment.

Psychological effects are often conditional on a specific interpretation of the stimulus, manipulation, or dependent variable in the study. For example, performance-approach achievement motivation (Elliot, 1999) can only predict school grades if participants in the study perceive grades as a valuable achievement. Empathy can only facilitate a helping behavior if the target behavior is recognized as, indeed, helpful. The interpretation of the studied phenomenon is then a boundary condition for the studied effect: If it remains similar between the contexts of the original study and its replication, the effect stands a chance of replicating; if it is sufficiently different, the effect cannot be replicated.

Intuitively, some interpretations are less generalizable than others. Getting good grades on a math exam will probably be interpreted as an achievement by many participants across cultural and historic contexts and will be positively related to achievement motivation. Getting good grades in an art class may be affected by achievement motivation for some participants and in some contexts, but not in others. Most people would recognize helping a victim of a physical attack as helping behavior, but helping a classmate cheat on an exam might be considered helpful by some people in some cultural and historic contexts, but not by others - and this helping behavior might not be as affected by empathy towards the beneficiary. The Van Bavel et al. (2016a) study indicates that context dependence can be inferred by experts with at least some accuracy - in their study, the experts' ratings of context sensitivity were significantly associated with the chance of studies to replicate.

To advance from informal judgments towards formal, testable predictions of whether design elements of a study hold the same meaning across contexts and individuals, one would require a theoretically grounded and quantifiable construct that reasonably corresponds to "interpretation" or "meaning" in research applications.

Such a construct would allow explicating assumptions about interpretations of studied phenomena that may affect the generalizability of psychological studies. Beyond generalizability concerns, unstated assumptions about interpretations or "meanings" of design elements may lead to loss of power of psychological models. If participants have to interpret a design element in a particular way for the effect to appear, and if participants differ in the extent to which they make that interpretation, the size of the effect will be underestimated. Consider a hypothetical study where grades in an art class are predicted from achievement motivation. The implicit assumption in this study is that for participants, getting a good grade in an art class represents a meaningful achievement. If for some of the participants that assumption is violated-they do not think the grade for the art class is important, or even think that being good at art is looked down upon-the relationship will not hold for these participants or may even take the opposing direction. Failing to differentiate between participants who conform to the assumption and those who violate it would then lead to an underestimation or even 'canceling-out' of the effect.

Attempts to quantify "meaning" in psychology have been few. Writing in 1957, Charles Osgood and colleagues list the following reasons for the general lack of interest in the task: 1) the similarity of "meaning" to nonmaterial abstractions such as "idea" and "soul", criticized by J. B. Watson as "ghosts" of psychology; 2) the implicit nature of meaning; and 3) the notion that meanings are infinitely variable, making them resistant to systematic assessment (Osgood et al., 1957). Osgood et al. conclude that the task of theorizing about meaning is largely ignored by psychologists and left to deal with for philosophers-which is still true today.

In this paper, I aim for the next best thing to a measure of meaning - a quantifiable construct that covers a significant proportion of what we called "meaning" and "interpretation" earlier, and that can be used to address practical problems outlined in this introduction.

\section{Meaning and intention}

As Osgood and colleagues noted, psychologists defer to philosophers to theorize about meaning. There are more theories of meaning in philosophy than this paper allows to cover in detail, but they can be broadly classified into two types: semantic and foundational theories (Speaks, 2019). Semantic theories of meaning are concerned with the meaning of language and the way in which semantic content is assigned to linguistic expressions. These theories address the question "what is the semantic value of an expression". In contrast, foundational theories of meaning aim to ex- 
plain how expressions come to have semantic meaning. These theories address the question: "by virtue of which facts does the expression have this semantic meaning". Since the task at hand is capturing contextual differences in the meaning of experimental manipulations, dependent variables, and psychological situations, foundational theories are more relevant.

One of the central problems faced by foundational theorists of meaning is that the same semantic expression can take different meanings. For example, the utterance "Bremen is not California", depending on the context, may mean that the German city of Bremen is not located in California, that the weather in Bremen is much worse than it is in California, or that the laws and customs of California are not observed in Bremen. The problem is analogous to the one we are trying to address - that the meaning of formally identical manipulations, behaviors, and other study elements may vary across contexts.

In his influential meaning project, Paul Grice (1957, 1968) argued that the meaning of a semantic expression can be defined in terms of "speaker meaning", or what the speaker means by making the expression. The "speaker meaning", in turn, can be defined in terms of the intention of the speaker. Reusing the previous example, "Bremen is not California" is an expression uttered by a speaker for some purpose, and the meaning of the expression depends on that purpose. In a similar vein, in the discussion of thick and thin descriptions, Geertz (1973) argued that to understand the meaning of behavior in context one has to examine not the objective properties of the behavior, but the intention with which it is performed.

Without taking a stand on whether the construct of intention is sufficient to define meaning, let us examine whether intention can serve as a substitute for meaning for our particular practical problem. Rephrasing the examples from the introduction, it can be said that achievement behaviors are behaviors performed with the intention to achieve, and helping behaviors are behaviors that are performed with an intention to help. The intention, in turn, can be defined in terms of expected consequences. Striving for a good grade in an art class is an achievement behavior insofar that it is expected to result in a demonstration of competence and be appreciated by others. To that extent, we would expect it to constitute a valid approach-performance achievement goal. If it is done with other outcomes in mind-for example, to qualify for an art camp, to spend time with a friend, or with no particular expectation, as a by-product of a genuine pleasure taken in art-we would not expect this type of achievement motivation to affect it. Similarly, helping a classmate cheat on a test would be a helping behavior if it is performed to provide valuable outcomes for another person-and then we would expect it to be governed by psychological mechanisms that underlie prosocial behavior. If, on the other hand, it is done with the inten- tion to avoid confrontation with a bully, this would not be the case. The same behavior performed to achieve qualitatively different outcomes can be said to have different meanings.

After this rephrasing, we are left with an easier task: to formalize and quantify perceived consequences of psychological phenomena, such as actions or events, to approximate the differences in meanings of these phenomena across individuals and cultural and historic contexts.

\section{Perceived Consequences of Behavior}

"Perceived consequences of actions" are better represented in psychology than "meaning", although they are rarely used to capture the latter. Perceived consequences of actions are an element of dominant theories in social and personality psychology, such as the Theory of Planned Behavior (TPB; Ajzen, 1985) and the Cognitive-Affective System Theory of Personality (CASTP; Mischel \& Shoda, 1995). In these theories, perceived consequences of actions play a crucial role: They are the cognitive grounds for evaluation of behavior. In TPB, perceived consequences of actions are incorporated into a broader category of "behavioral beliefs" and are theorized to contribute to the attitude. Behavioral beliefs (alongside normative beliefs and control beliefs) receive less attention in empirical research than the more direct predictors of behavior: attitudes, norms, and perceived behavioral control (Ajzen, 1991). CASTP describes beliefs about outcomes of behavior as one of the five "cognitive-affective units" and explicitly points to individual differences along this dimension. However, individual differences in expectancies and their effects on behavior also generated little research within this paradigm. Differences in the content of expectations have recently received attention in behavioral economics (Coibion et al., 2019, D'Acunto et al., 2019a, 2019b), but such models are currently uncommon in psychology.

Two circumstances may explain the inconsistency between the importance of the content of perceived consequences of behavior for psychological theories and the relative scarcity of empirical research on the subject. First, possible consequences of behavior appear infinitely variable, making measurement and comparison practically difficult. And second, researchers may underestimate the variability in the perceptions of behavior on part of their research participants due to lack of direct contact with participants and "naïve realism" of the researchers, or tendency to believe that their view of reality is objective (Ross \& Ward, 1996), and that it is widely shared (Ross et al., 1977).

\section{Expectancy}

A cluster of theories that deal with perceived consequences of action most directly is commonly referred 
to as the Expectancy-Value Theory (EVT; Ajzen \& Fishbein, 1975, Edwards, 1954; Rosenberg, 1956). Similar models have been proposed by multiple researchers, but the unifying theme of EVT models is that an evaluation of a behavior, object, or event can be described as a product of expectancy (probability of an outcome occurring) and value assigned to that outcome.

Specific definitions of the expectancy and value terms vary across models, but the principal equation can be summarized as follows:

$$
A_{o}=\sum_{i=1}^{N} E_{i} V_{i}
$$

Where $A_{o}$ is the attitude towards an object $O, E_{i}$ is the expectancy term for some outcome $i, V_{i}$ is the evaluation of $i$, and $N$ is the number of outcomes of object $O$.

Proposing future directions for research in EVT, Feather (1988) argued that a distinction should be made between different types of expectancy term: the one that refers to the probability of the behavior or event occurring, and the one that refers to the probabilities of qualitatively distinct consequences of the behavior or event occurring. This is an important distinction since only the second kind of the expectancy term can potentially capture the qualitative differences in perceived consequences of behavior that I describe above.

Several formulations of EVT incorporated the idea that the same behavior, event, or object can be evaluated on multiple grounds (Ajzen \& Fishbein, 1975, Eccles et al., 1983, Rosenberg, 1956), and that resulting evaluations jointly inform the attitude. Practical applications of EVT, however, often rely on an expectancy term that refers to the probability of the event occurring (see, for example, research on expectancy-value models in education based on Wigfield and Eccles's (2000) account of achievement motivation). Generalizability and applicability of EVT-like models that incorporate multiple grounds of evaluation depend on a typology of grounds for evaluation that has to be 1) comprehensive, i.e. describe a large subset of the universe of possible bases of evaluation; 2) universal, i.e. applicable across situations cultures, times, and individuals and 3) discriminant, i.e. capable of differentiating bases of evaluation in respect to content.

There have been attempts to classify grounds for evaluation of objects. Osgood and colleagues (1957) sampled a list of evaluative words from Roget's thesaurus (Roget, 1878) and performed a factor analysis on them that resulted in a list of eight semantic dimensions, of which three (evaluation, potency, and activity) displayed acceptable generalizability to other modalities. This list, however, has a low capacity to discriminate grounds of evaluation based on content due to the abstractness of its categories. Fishbein (1975) theorized that an attitude towards an object is determined by the associations of that object with an unlimited universe of other objects, and by the evaluations of these associated objects. Fishbein did not try to systematize this universe of possible connections, resulting in a comprehensive conceptualization that is impossible to apply. Eccles (1983) proposed the following typology of evaluative components: attainment value or importance for self, intrinsic value or enjoyment and interest, utility value or usefulness for other goals, and cost for time and psychological and physical resources. This typology is both universal and comprehensive but, just as Osgood's dimensions, does not allow to discriminate between qualitatively different grounds for evaluation. For example, a person may pursue a career as a firefighter because they see it as an opportunity to help people, while another person may be motivated by an opportunity to display courage and mastery. According to Eccles' typology, both people would attribute high "attainment value" to becoming a firefighter but have qualitatively different motives. Finally, Rosenberg (Rosenberg, 1956) used personal values as grounds for evaluation. In his study, he relied on then-contemporary theories of values to produce a list of 35 value items and used ratings of value importance and perceived consequences for each of the values to predict an attitude towards a behavior, "allowing Communists to address the public". Rosenberg's list allowed discriminating between qualitatively different grounds for evaluations - his theorizing suggested that some people could approve of allowing the Communists to spread their ideas on the grounds of it helping "people be well-educated", while others could approve of it on the grounds of promoting "open expression of disagreement between people". Rosenberg made no argument as to the universality of his list or its comprehensiveness.

Since these attempts were made, research on values in psychology has significantly advanced. The Theory of Basic Human Values (Schwartz, 1992) provides a typology of grounds for evaluation that satisfies the three requirements outlined above: comprehensiveness, universality, and discriminant capacity. Building on earlier accounts of human values (Kluckhohn, 1951; Rokeach, 1973), Schwartz defines values as beliefs that refer to desirable goals or end-states, transcend specific situations, serve as standards or criteria for evaluation of actions, objects, and events, and that are ordered by relative importance (Schwartz, 1994a). Schwartz argues that values are cognitive representations of universal human needs that are grounded in our evolutionary history: the biological needs of organisms, the need for survival and welfare of social groups, and the need for coordinated social interactions (Schwartz, 1992).

Taking an empirical approach, Schwartz described a system of dynamic relations that underlies the endorsement of values. He found that the organization 
of human values can be summarized with a circular structure, where values located near each other (and for which endorsement correlates most positively) express similar motivational goals, and values located opposite to each other (and for which the endorsement correlates least positively) express competing motivational goals. This circular structure can be further summarized with two dimensions. The first dimension, labeled "openness to change vs conservation", juxtaposes values that represent the needs for independence, exploration, stimulation, and learning with values that represent the needs for safety, order, and predictability. The second dimension, labeled "selfenhancement vs self-transcendence" contrasts values that represent concern for the outcomes for the self with values that represent concern for the outcomes for others. Along the two dimensions, the original theory (Schwartz, 1992; Schwartz \& Bilsky, 1990) places 10 (Table 1), and the revised theory (Schwartz, 2017) - 19 basic human values.

Schwartz's typology of values is comprehensive: any value could be positioned in the circular structure based on its relations to other values. The boundaries between values are arbitrary and are introduced largely for the purpose of measurement. The theory of basic human values describes a universal structure of value relations; the original study (Schwartz, 1992) evaluated the value structure using 40 diverse samples from 20 countries, and further studies established crosscultural validity of the values structure (Cieciuch et al., 2014; Davidov et al., 2008). Finally, Schwartz's theory allows discriminating grounds of evaluation based on their content because values are defined in relation to qualitatively distinct motivational goals that they serve. For example, a person who wants to pursue a career in firefighting to help people would have a positive evaluation of this career option based on self-transcendence values, while a person who is attracted to firefighting because of the opportunities to express courage and skill would base their evaluation on openness to change or self-enhancement values.

I propose that perceived consequences of actions, objects, and events for values can serve as a practical substitute for the construct of "meaning" for theorizing about and quantifying "meaning-related" boundary conditions for a broad subset of psychological effects.

\section{Specification of VIB}

\section{Assumptions}

At this point, I will formalize the assumptions that are inherent in the argument presented above and that I will continue to rely upon further in this paper.

1. People's subjective interpretation of reality, rather than objective reality, guides their attitudes and actions.
2. People use values as grounds for evaluation of actions, objects, and events.

3. People's perceptions of the consequences of actions, objects, and events for values may differ across individuals, situations, societies, and times.

\section{Definition}

To capture the variation in perceived consequences of actions, objects, and events for values I propose the construct of a VIB. VIBs are personal beliefs about the consequences of actions, objects, or events for values. Examples of VIBs are "sharing toys is kind", "drugs are dangerous", and "being invited to such and such conference is prestigious".

VIBs are beliefs, meaning they are dispositions to assert the truthfulness of a proposition (Schwitzgebel, 2002), in this case, that "object A has positive or negative consequences for value B". The strength of the VIB would be a function of the perceived likelihood of the consequences occurring (Easwaran, 2011) and the severity of the consequences. VIBs are personal beliefs, meaning they are held by individuals, as opposed to groups of people. Finally, VIBs encompass propositional beliefs that posit a relationship between an object and a value. For example, "sharing toys is kind" is a proposition that asserts that an action (sharing toys) promotes the attainment of a value (kindness), and that can be rephrased as "sharing toys promotes the attainment of kindness".

A set of VIBs comprised of distinct VIBs for each of the values in a typology exhausts the possible consequences of a specific object for values. An individual VIB can be positive or negative (a person can believe that snakes are dangerous or that they are safe). VIBs can also differ in strength (a person can believe that snakes are very dangerous or only somewhat dangerous).

Further, I will describe the relations between VIBs and related constructs: attitudes, values, and social representations.

\section{Conceptual Distinctions}

\section{Attitude}

VIBs are closely related to attitudes. Attitudes are dispositions to evaluate an object positively or negatively (Eagly \& Chaiken, 1993), while VIBs are dispositions to evaluate an object as having positive or negative consequences for a value. Both constructs describe dispositions, meaning they are not "stored" or otherwise directly represented in a person's mind, but are rather consistently produced in response to a situational demand (Schwitzgebel, 2002). For example, when asked, "What attitude do you have towards raspberry pizza?" a person would be able to answer even if 
they never thought about this subject before. In a similar way, a person can say if they consider raspberry pizzas as bland or daring, pleasant or unpleasant, conventional or unconventional without having to retrieve a previously formed belief from their memory.

VIBs differ from attitudes in their specificity - while an attitude is a summary judgment of an object, the VIB is a judgment on the basis of a specific criterion a value. To illustrate the difference, consider two people with a strong positive attitude towards apples. The first person can like apples for their taste - or, in other words, believe that apples have positive consequences for the value of hedonism. The second person might not care for the taste of apples but believe that apples help them stay healthy - or that apples have positive consequences for the values of health and personal security. In this scenario, both persons hold the same attitude, but it is supported by perceived consequences for different values (see also Ajzen \& Fishbein, 1975. p. 223).

\section{Value}

VIBs are functionally distinct from values: values serve as both goals and grounds for evaluation of objects (Schwartz, 1994a), while VIBs are the results of value-based evaluations that inform attitudes and provide means for value attainment. Values are universal - they are cognitive representations of evolutionarily significant needs that are common to all humans. Both genetic and environmental factors contribute to the development of personal value hierarchies (Knafo \& Spinath, 2011; Uzefovsky et al., 2016). VIBs, on the other hand, are not universal and are exclusively products of learning and socialization.

VIBs are more specific than values: values are important goals, guiding principles in a person's life that transcend specific situations. For example, a person for whom the value of security is very important would consider safety an important goal at home, while traveling, and on vacation. In contrast, a valueinstantiating belief is specific to a particular object: a person might consider a game of tag dangerous but have no such belief towards the game of hide-and-seek. VIBs, then, are located lower in the cognitive hierarchy than values (Maio, 2010).

\section{Social Representation}

VIBs aim to capture differences in perceptions of an object in context. In that respect, they serve similar functions as social representations (Moscovici, 1961. 1984). A social representation is the "stock of ideas", a system of beliefs, values, ideas, and practices that pertain to a specific social object and are shared among members of a group for the purpose of acting towards the object and communicating about it (Moscovici, 1961, 1984). VIBs, however, are more narrowly defined than social representations and differ from them in several important ways.
First, VIBs, compared to social representations, are limited in content: they describe only content relevant to a specific value. For example, a social representation of vaccination would encompass all beliefs, values, ideas, and practices related to vaccination in a specific community. A security VIB about vaccination, on the other hand, would be limited to perceptions of vaccinations as safe or unsafe. A universalism VIB would reflect the degree to which a person sees vaccination as benefiting the vulnerable members of society, a hedonism VIB would describe the degree to which vaccination is seen as pleasant or unpleasant, and a conformity VIB would describe the degree to which vaccination would conform to or violate perceived expectations of others.

Second, a VIB represents a single judgement and can be comprehensively described by its valence and strength. Social representations refer to the entirety of ideas about an object in the society, varying both in valence and in relevance for on behavior. For example, a social representation of vaccination in a community may include both pro-vaccination and anti-vaccination discourses, but an individual can hold only one security VIB for vaccination: they can believe it to be either safe or unsafe. This belief may be informed by some elements of the social representation of vaccination in the community but is distinct from the social representation.

Finally, a VIB is a personal belief. Similar to a value or an attitude, it is located within the mind of an individual. A social representation, on the other hand, is a feature of a society. For that reason, VIBs are better suited for describing individual-level differences in perceptions of social objects and distributions of such perceptions within a group.

\section{Consequences and Antecedents of VIBs}

VIBs are beliefs that posit a relationship between an object and a value - they are cognitive "bridges" that link evolutionarily-grounded motivations (as expressed in values) to particular actions, objects, and events situated in a particular environment. The role of such beliefs can be explicated with a thought experiment-let us look at the environment we are currently in (a room, an office, a café), and scan it for objects. Once we find one, let us strip it from all value-relevant, abstract meaning: let us imagine that it is neither comforting nor exciting, does not serve or preclude our success, is neither beneficial nor harmful for others, not safe and not dangerous. As we do this, we will likely find little reason to think about it at all-if it has no implications for abstract goals that we find important, it itself is unimportant and does not deserve approaching or avoiding.

The experiment illustrates the motivational implications that set VIBs apart from other kinds of beliefs. VIBs are a necessary precondition for value-driven action-a value can only motivate the choice of an ac- 
tion if behavioral options vary in the perceived consequences for the value. Additionally, salience and availability of VIBs conditions their effect on behavioral choices. Value-driven action comprises a substantive subset of action: values are often invoked effortlessly in judging behavioral options and affect attention, perception, and emotional responses when planning action (Hitlin \& Piliavin, 2004, Rohan, 2000). Since VIBs enable value-driven action, they are a predictor of attitudes and consequent affective responses and actions.

How are VIBs acquired? VIBs are a type of belief and are acquired through the same means as other beliefs: personal experience, inference, and socialization (Wyer \& Albarracin, 2005). For instance, a person may come to hold a belief that dogs are dangerous (a negative VIB linking dogs to the value of security) through personal experiences-after being bitten by a dog; through inference - after being bitten by another carnivore and concluding that all carnivores are dangerous, dogs are carnivores, and therefore dogs are dangerous; or through socialization-after being told that dogs are dangerous by another person.

Given these routes of belief formation, individualand group-level differences in VIBs could be predicted from systematic differences in personal experiences, inferences, and socialization. Although VIBs have not been measured explicitly in earlier studies, we can make inferences about differences in VIBs from attitudes: between-individual differences in attitudes imply differences in the direction (positivity) and intensity of underlying VIBs.

\section{Personal Experience}

An example of influence of personal experience on VIBs could be found in the literature on the contact hypothesis in intergroup relations (Pettigrew, 1998): individuals who had positive personal experiences with outgroup members tend to have more positive attitudes towards outgroups. This relationship is mediated by perceptions of consequences that outgroup members have for valued outcomes. In a study of anti-Muslim attitudes in the Netherlands, for instance, friendship with members of ethnic minority members negatively related to responses to items such as "Dutch people get fired because of minorities" and "Minorities threaten Dutch culture", which, in turn, were positively related to anti-Muslim attitudes (Savelkoul et al., 2011).

\section{Inference}

Individual circumstances can also provide different opportunities for inference. Research in extended contact effect, for example, shows that knowledge of an in-group member being friends with an outgroup member has a positive effect on the attitude towards outgroup members (Wright et al., 1997); In this case, while not having personal experience with outgroup members, people can infer that interaction with outgroup members may bring about positive consequences.

\section{Socialization}

Often, beliefs are acquired through communication (Wyer \& Albarracin, 2005) — for many objects, our personal experience is insufficient to ground a belief about their consequences. In the earlier example, most Dutch people expressed agreement or disagreement with the statement "Dutch people get fired because of minorities" - while most of them, in all likelihood, did not have a personal experience of being fired because of minorities. Systematic differences in socialization experiences-differences in exposure to media, political affiliation, educational curriculum, gender roles - are likely to lead to acquisition of different VIBs.

Like other beliefs, VIBs are learned-people are born without expectations of particular outcomes of objects in their environment. As they mature, they learn to interpret the environment in terms of abstract goals and values. A toddler looking at Kandinsky's paintings sees a senseless assembly of bright shapes, identical in meaning and value to a painting by Still, Pollock, or a bright packaging of a toy. As a person accumulates experience in interacting with art and communicating about art, they might find Kandinsky's art daring or boring, stimulating or soothing, insolent or respectful. Such learned individual perceptions would direct their behavior towards Kandinsky's paintings and differentiate it from behavior towards paintings by Pollock and toy boxes. People learn to relate to most other objects in a similar way, starting from scratch and forming their beliefs over a lifetime, learning about consequences of outgroup members, helping behaviors, different types of work and leisure, and so on for valued goals. The objects in the environment then become envaluated - imbued with value-relevant expectations. This process links socialization experiences to behavioral dispositions, and specific VIBs act as mediators of the relationship between personal experiences and socialization on the one hand and attitudes and behavior on the other.

\section{Implications}

\section{VIB as a Boundary Condition}

Above, I presented a value-based model of attitude that uses basic human values (Schwartz, 1992) as grounds for evaluation and perceived consequences for values as expectancies. The model can be summarized as follows:

$$
A_{o}=\sum_{i=1}^{N} V I B_{i} V_{i}
$$

Where $A_{o}$ is the attitude towards an object, action, or event $O, V I B_{i}$ is the value-instantiating belief about 
consequences of object $O$ for value $i, V_{i}$ is the importance attributed to basic human value $i$, and $N$ is the number of basic human values. This formulation has the following benefits: first, $N$ (or the number of grounds for evaluation) is an imagined quantity in classic formulations of EVT (Ajzen \& Fishbein, 1975; Edwards, 1954), while here it refers to the finite number of basic human values: 10 in the original (Schwartz, 1992), and 19 in the refined (Schwartz, 2012) version of the Theory of Basic Human Values. Second, by the merit of using universal human values as grounds for evaluation, it is applicable across evaluated objects, as well as people making the evaluation and situations, cultural and historic contexts within which the evaluations are made. And finally, this theoretical model allows differentiating between qualitatively distinct motives underlying appraisal of an object.

This formulation provides a theoretical and practical tool to hypothesize and test boundary conditions that relate to the "meaning" of actions, objects, and events in context. There are two ways in which VIBs can bound psychological effects: through lack of VIBs and through conflicting VIBs. From the equation above, when an object is not envaluated (i.e., is not expected to have either positive or negative consequences for a value), the VIB term of the equation takes the value of zero, and the attitude is independent of the value or its underlying motivational goal. For example, for people unfamiliar with the concept of vaccination, a flu shot is neither a safe nor an unsafe event, and the value of security will not motivate the attitude towards vaccination. Once a person learns about vaccination and develops expectations for its consequences, the desire to vaccinate can be affected by their concern for personal security and other factors, such as risk aversion or reactance. In addition, if there is a population in which both negative and positive VIBs are represented, the same value can motivate opposing attitudes in the corresponding sub-populations. For example, if half of the population beliefs that vaccinations are safe, and half beliefs that they are unsafe, the value of security can motivate approval of vaccinations for one half of the sample, and disapproval for the other half. As a result, attempting to estimate the effect of the value of security (or risk aversion, or reactance) on the desire to vaccinate will result in an underestimation or "canceling out" of the effect altogether.

\section{VIBs Bound the Effects of Values}

The effects of a value on the attitude and behavior depend on whether the action, object, or event in question promotes or hinders realization of the motivational goal that underlies the value (Schwartz, 1996). If an object promotes the attainment of a value, it can be hypothesized that importance attributed to the value would be positively related to the attitude towards that object. If an object hinders the attainment of a value, a negative relationship is hypothesized. Yet, differences in perceived consequences of actions and objects for values are not typically incorporated into models that predict attitudes and behaviors from values.

As discussed above, contextual differences in personal experiences, inferences, and socialization can result in differences in perceived consequences of actions and objects for values. Such differences would cause contextual variation in value-attitude and valuebehavior relations. The literature provides plentiful examples of contextual variation in value-attitude relations. The relationship between religiosity and the value of conformity is more positive in countries with more harmonious relations between church and state (Roccas \& Schwartz, 1997), conservative values are associated with right-wing political orientation in western countries, but the link is weakened or even reversed in countries with communist past (Barni et al., 2016, Piurko et al., 2011), the conservation-openness value dimension is negatively related to attitude towards cultural diversity among majority group members, but positively - among minority group members (Grigoryan \& Schwartz, 2020).

Interpreting the observed variations in value effects, researchers point to differences between the studied groups in meaning ascribed to the object under study (e.g., Barni et al., 2016; Grigoryan \& Schwartz, 2020, Piurko et al., 2011)), the nature of the object (e.g., Roccas \& Schwartz, 1997), or features of the ideologies pertaining to the object (e.g., Barni et al., 2016). Rephrasing such interpretations using the narrower construct of VIB allows being more specific in the descriptions of differences between contexts, generating testable predictions corroborating the interpretations, and developing new hypotheses. Taking the churchstate relations study (Roccas \& Schwartz, 1997) as an example, the relationship between conservation values and religiosity can be expected to be more positive in countries where people hold more positive security and conformity VIBs about religion and religious practices-in other words, where people believe that practicing their religion is safer and conforms to expectations of others more. This is a testable interpretation that can be validated by asking people about their perceived consequences of religious practices. Further, we can hypothesize about the generalizability of the effect of conservation values on religiosity to other contexts: for example, we may expect this effect to not generalize to members of religious minorities, since they may believe that practicing their religion has less positive consequences for the value of conformity; or to situations with salient discrimination or persecution on religious grounds, where people may believe that practicing their religion can have negative consequences for their security.

The role of VIBs as an individual-level moderator of value-attitude and value-behavior relation that may account for differences in contextual "meaning" of behavior has been corroborated in two studies. The first 
study (Ponizovskiy, 2020) was a survey of political attitudes and intentions in a sample of 2038 US residents, with liberal and conservative participants equally represented. The study included four behaviors: voting for Donald Trump in 2020 presidential election, voting for Bernie Sanders in the same election, donating to a pro-immigrant charity, and supporting an anti-abortion bill. The study documented differences in VIBs between liberal and conservative participants for all four behaviors: for example, while liberals saw voting for Trump mostly in terms of negative consequences for the value of universalism, conservative participants saw this action in terms of positive consequences for self-direction and security. The variance in VIBs predicted differences in strength and direction of value-behavior effects, with significant interactions observed for all four behaviors. Finally, incorporating differences inVIBs into the model substantially improved its predictive power. In the second study (Ponizovskiy et al., 2019), VIBs were manipulated; three actions were presented in vignettes that portrayed them as either safe or unsafe (positive vs. negative security VIB), promoting or thwarting social justice (positive vs. negative universalism VIB), and conformist vs. non-conformist (positive vs. negative security VIB). In all three trials, the VIB moderated the relationship between the values and the intention to perform the action. The direction of the relationship was determined by the VIB, so that if a behavior was portrayed as safe, it related to the value of security positively, and if it was portrayed as unsafe, the relationship was negative. Together, the studies support the role of the VIB as the necessary precondition of the value-behavior relationship, illustrate that VIBs towards the same object may vary across individuals and groups, and indicate that VIBs lend themselves to both measurement and manipulation.

\section{VIB as a General Boundary Condition}

The previous section focused on the use of the VIBs as a proxy for meaning to account for contextual differences in value-attitude and value-behavior effects. However, contextual differences in meanings do not only limit the generalizability of the effects of values, but also the generalizability of other effects that rely on assumptions of a particular appraisal of an object. Such assumptions are commonplace: models that predict helping behavior often assume that the behavior under study is interpreted as helpful (in other words, that participants have positive benevolence VIB towards it); models that predict academic achievement often assume that outcome measures are interpreted by participants as achievements (or that participants have positive achievement VIB about them). As theorized above, and as first evidence indicates, VIBs may differ across contexts and individuals: an outcome that is seen as an achievement in one context or by one individual might not be interpreted the same way in an- other setting. This can lead to a failure of the effect to replicate with a different set of participants. Explicating assumptions about the relevant VIBs can help theorize about limits to generalizability of such effects, test such boundary conditions, and improve the predictive power of psychological models.

It is beyond the scope of the present paper to estimate the proportion of the psychological effects that are bound by the "meaning" of the attitude or behavior under study. To demonstrate that such effects are not uncommon, I will present two examples from the most recent issue of the European Journal of Social Psychology, 50(3).

In Study 2 from Gorska et al (2020), a model is tested in which solidarity-based action (participation of men in a march against abortion ban) is negatively predicted from collective narcissism of participants, and the effect is mediated by empathy towards women. The data agree with the theoretical model. The assumptions that underly the theoretical model are that 1) women are perceived by the male participants as an out-group that requires support, and 2) that resisting an abortion ban is a form of such support. In other words, the assumption is that the behavior that is being predicted has positive universalism VIB-it is perceived as having positive consequences for the values of social justice, tolerance, understanding and appreciation of all people. While this assumption is justified for many contexts, and evidently held true for the context under study, stating it explicitly allows us to theorize circumstances in which the observed relationship might not hold. For example, people might not believe that protesting against an abortion ban has positive consequences for universalistic values if they are strongly socialized within the Catholic church, as the Catholic church affirms that abortion is a moral evil and a threat to the woman's religious salvation. If a sample is comprised of highly religious Catholics, participation in an abortion ban march would unlikely be influenced by collective male narcissism or empathy towards women. Participation in a march that opposes abortion bans on grounds other than women's rights-for instance, on grounds of individual freedom or anti-religious sentiment-is also unlikely to be motivated by empathy towards women. On the other hand, the universalistic interpretation of collective action against abortion laws is likely to be made by people who have experience with the women's rights movement, such as university students from around the world.

Similarly, in Study 1 from Renger et al. (2020), self-respect as a Muslim predicted intention for normative protest in response to injustice in a hypothetical employment situation. The authors theorize that self-respect predicts assertive responses to injustice. The underlying assumptions are that the hypothetical situation is perceived as unjust (participants possess negative universalism VIB towards the situation), 
and that the normative reactions-objecting, contacting a union, and suing - are seen as rectifying the injustice (participants possess positive universalism VIB towards these actions). Again, we can expect the theorized relationship not to generalize to scenarios where people do not hold these VIBs-for example, where people do not trust the judicial system and the labor unions, or that have little exposure to antidiscrimination discourse.

These are but few examples, but they represent a typical scenario in social psychological research: application of general psychological theories to particular social realities. In doing so, researchers necessarily make assumptions about the 'meanings', or expectancies of consequences of actions, objects, and events in the context that they study. Such assumptions might hold for some contexts, but not for others, making it more difficult to generalize psychological knowledge and develop a universal account of human psychology. The construct of VIB helps to explicate assumptions inherent in such applications and theorizing about limits to generalizability of observed effects.

\section{Other Implications}

A VIB is a narrowly defined, quantifiable operationalization of meaning. Differences in meaning, or interpretations of objective reality, limit the generalizability of psychological findings, and VIBs help to theorize about and test such limitations. Yet differences in meaning do not only limit generalizability of psychological effects - they are also an integral part of many phenomena and processes that interest psychologists. VIBs can be used as a practical substitute for meaning in these applications as well. Below, I briefly discuss some of these applications and propose testable hypotheses based on the VIB construct.

\section{Cooperation and Intergroup Relations}

Shared VIBs represent compatible interpretations of reality that are required for many types of cooperative action. Consider a group of students preparing for a difficult test. If all students believe that cheating would devalue their achievement, they can expect each other to behave honestly on the test. If all students believe that cheating can help them achieve by increasing their grade, they can expect each other to not disclose cheating to the teacher, and to help each other cheat. If, however, some students believe that cheating is detrimental to achievement, while others believe it to be beneficial, cooperation among them becomes impossible. Brewer and Caporael (Brewer \& Caporael, 2013) argue that trust and predictability form the evolutionary basis of intergroup bias - we prefer to cooperate with ingroup members because we can better predict what they will do. In line with this argument, I hypothesize that sharing VIBs with outgroup members leads to weaker intergroup bias, while holding opposing VIBs leads to stronger intergroup bias and can result in conflict. VIBs are acquired through personal experience, inference, and socialization; groups that have strong institutions that facilitate socialization among their members, and the members of which share personal experiences-such as religious and ethnic groups - are then likely to have lower within-group and higher between-group variance in VIBs. Further, I expect groups that are more homogenous in VIBs to be perceived as higher in entitativity, or the degree to which they are perceived as coherent entities.

VIBs can be used to model the processes of acculturation and intercultural communication. A person holding VIBs that are not shared by members of a host society or an interlocutor may lead a person to make inaccurate predictions of other people's actions, miss opportunities for cooperation, and be sanctioned for their actions. Over time, shared experiences and opportunities for socialization can bring the VIBs of people from different backgrounds closer together. I therefore expect VIBs of immigrants to become more similar to those of the host society over time, and the VIB congruity to predict positive outcomes for immigrants, such as the size of social networks, employment, mental health, and well-being.

\section{Attitude Formation and Persuasion}

As VIBs contribute to cognitive grounds of the attitude, the conceptualization is relevant to the processes of attitude formation and change. Via values, VIBs link actions, objects, and events to evolutionarily significant needs. Information that can inform VIBs should be then perceived as relevant to motives with survival value, and should be prioritized at the stages of perception and processing. I expect people to attend to information about consequences for values more than to information about other types of consequences. When forming attitudes, I expect VIB-relevant information to have a stronger effect on the attitude than VIB-irrelevant information. Furthermore, I expect that for an individual, information supporting the VIBs for values that are high in their personal hierarchies will have a stronger effect on the attitude than information supporting the VIBs for values that are low in their personal hierarchy.

\section{Culture}

Clifford Geertz (1973) has famously defined culture as "webs of significance", the network of meanings that orders and directs the behavior of people in context. Geertz argued that understanding the contextspecific functions that actions serve is more informative of culture than describing the actions themselves. While this view of culture is influential among anthropologists, it is not well-represented in psychology. Psychological accounts of culture are dominated by dimensional theories (e.g. Hofstede, 1980; Inglehart, 
2015: Schwartz, 1994b) that order countries along highly abstract dimensions rather than provide detailed descriptions of the content of cultural learning. VIBs provide an opportunity to assess and compare functions and meanings of actions in cultural contexts. Differences in functions of behaviors across cultures are captured by the construct of functional (in)equivalence (van de Vijver \& Tanzer, 2004). A classic example of lack of functional equivalence is pet ownership: while dogs and cats are treated as family members in the US, they are considered a nuisance in many other countries. We can then expect actions that improve the well-being of dogs and cats to have stronger positive benevolence VIBs in the US compared to countries where dogs and cats are not considered pets. VIBs can also be used for detailing the mechanisms proposed by evolutionary models of culture. VIBs link actions to evolutionarily grounded motives, which makes them likely candidates to play a role in behavioral adaptation to the environment. Evolutionary models of culture, such as the parasite stress model (Fincher \& Thornhill, 2012), lack an observable link between the environmental pressures and highly abstract cultural indices, such as Hofstede's cultural dimensions, on the one hand, and specific actions, such as prayer, on the other. VIBs can act as a gauge for context-specific "ideas, ways of thinking, and attitudes" (Fincher \& Thornhill, 2012, p.63) that are selected under evolutionary pressures. For example, we can expect negative security VIBs about actions that increase the likelihood of disease transmission to be more prevalent in environments with high disease stress.

\section{Conclusion}

In this paper, I presented the theoretical specification of VIBs, or beliefs about consequences of actions, objects, and events for values. VIBs capture the motivational meaning of objects by relating them to mental representations of evolutionarily significant needs. Using VIBs as a practical substitute for "meaning" can help us better theorize and test boundary conditions for psychological effects, improve the predictive power of psychological models, and provide new insights into topics that engage with the construct of meaning.

\section{References}

Ackerman, N., \& Paolucci, B. (1983). Objective and subjective income adequacy: Their relationship to perceived life quality measures. Social Indicators Research, 12(1), 25-48. https: //doi.org/10.1007/BF00428859

Ajzen, I. (1985). From Intentions to Actions: A Theory of Planned Behavior. In J. Kuhl \& J. Beckmann (Eds.), Action control: From cognition to behavior. (pp. 11-39). Berlin, Springer. https://doi.org/10.1007/978-3-642-697463_2
Ajzen, I. (1991). The theory of planned behavior. In Handbook of theories of social psychology (pp. 179-211). https : // doi .org/10.4135/ 9781446249215.n22

Ajzen, I., \& Fishbein, M. (1975). Belief, attitude, intention and behavior: An introduction to theory and research. Reading, MA, AddisonWesley. https://doi.org/10.2307/2065853

Barni, D., Vieno, A., \& Roccato, M. (2016). Living in a non-communist vs. in a post-communist European country moderates the relation between conservative values and left-right political orientation: A multilevel study. European Journal of Personality, 30(1), 92-104.

Brewer, M. B., \& Caporael, L. R. (2013). An evolutionary perspective on social identity: Revisiting groups. In M. Schaller, J. A. Simpson, \& D. T. Kenrick (Eds.), Evolution and social psychology (pp. 143-162). Madison, CT, Psychology Press. https://doi.org/10.4324/ 9780203782965

Cieciuch, J., Davidov, E., Vecchione, M., Beierlein, C., \& Schwartz, S. H. (2014). The cross-national invariance properties of a new scale to measure 19 Basic Human Values: A test across eight countries. Journal of Cross-Cultural Psychology, 45(5), 764-776. https://doi.org/ $10.1177 / 0022022114527348$

Coibion, O., Gorodnichenko, Y., \& Weber, M. (2019). Monetary policy communications and their effects on household inflation expectations. SSRN Electronic Journal. https://doi.org/10. 2139/ssrn.3316948

D’Acunto, F., Hoang, D., Paloviita, M., \& Weber, M. (2019a). Cognitive abilities and inflation expectations. AEA Papers and Proceedings, 109, 562-566. https://doi.org/10.1257/pandp. 20191050

D’Acunto, F., Hoang, D., Paloviita, M., \& Weber, M. (2019b). IQ, expectations, and choice (tech. rep.). Bank of Finland. www.suomenpankki. fi/en/research/research-unit/

Davidov, E., Schmidt, P., \& Schwartz, S. H. (2008). Bringing values back in: The adequacy of the European social survey to measure values in 20 countries. Public Opinion Quarterly, 72(3), 420-445. https :// doi .org/10 . 1093/ $\mathrm{poq} / \mathrm{nfn} 035$

Eagly, A. H., \& Chaiken, S. (1993). The psychology of attitudes. Harcourt Brace Jovanovich College Publishers.

Easwaran, K. (2011). Bayesianism I: Introduction and arguments in favor. Philosophy Compass, 6(5), 312-320. https://doi .org/10.1111/j . 1747-9991.2011.00399.x

Eccles, J. S., Adler, T. F., Futterman, R., Goff, S. B., Kaczala, C. M., Meece, J. L., \& Midgley, C. (1983). Expectancies, values, and academic 
behaviors. In J. T. Spence (Ed.), Achievement and achievement motivation ( $\mathrm{pp}$. 75146). San-Francisco, CA, W. H. Freeman.

Edwards, W. (1954). The theory of decision making. Psychological Bulletin, 51(4), 380-417. https://doi.org/10.1037/h0053870

Elliot, A. J. (1999). Approach and avoidance motivation and achievement goals. Educational Psychologist, 34(3), 169-189. https://doi.org/10. 1207/s 15326985ep3403

Feather, N. T. (1988). From values to actions: Recent applications of the expectancy-value model. Australian Journal of Psychology, 40(2), 105-124. https://doi . org/10.1080/ 00049538808259076

Fincher, C. L., \& Thornhill, R. (2012). Parasite-stress promotes in-group assortative sociality: The cases of strong family ties and heightened religiosity. Behavioral and Brain Sciences, 35(2), 61-79. https : // doi . org / 10 . 1017/ S0140525X11000021

Fishbein, M., \& Azjen, I. (1975). Belief, Attitude, Intention, and Behavior: An Introduction to Theory and Research. Reading, MA: Addison-Wesley.

Fraley, R. C., \& Vazire, S. (2014). The N-pact factor: Evaluating the quality of empirical journals with respect to sample size and statistical power. PLoS ONE, 9(10). https://doi.org/10. 1371/journal.pone.0109019

Francis, G. (2012). Publication bias and the failure of replication in experimental psychology. https: //doi.org/10.3758/s13423-012-0322-y

Geertz, C. (1973). The interpretation of cultures. Basic books.

Górska, P., Stefaniak, A., Malinowska, K., Lipowska, K., Marchlewska, M., Budziszewska, M., \& Maciantowicz, O. (2020). Too great to act in solidarity: The negative relationship between collective narcissism and solidaritybased collective action. European Journal of Social Psychology, 50(3), 561-578. https:// doi.org/10.1002/ejsp.2638

Grice, H. P. (1957). Meaning. The Philosophical Review, 66(3), 377-388. https://doi .org/10. $2307 / 2182440$

Grice, H. P. (1968). Utterer's Meaning, SentenceMeaning, and Word-Meaning Author(s). Foundations of Language, 4(3), 225-242. https://doi.org/https://doi.org/10.1007/97894-009-2727-8_2

Grigoryan, L., \& Schwartz, S. H. (2020). Values and Attitudes towards Cultural Diversity: Exploring Alternative Moderators of the ValueAttitude Link. Group Processes and Integroup Relations. https://doi.org/https://doi. org/10.1177/1368430220929077
Henrich, J., Heine, S. J., \& Norenzayan, A. (2010). The weirdest people in the world? Behavioral and Brain Sciences, 33(2-3), 61-83. https://doi. org/10.1017/S0140525X0999152X

Hitlin, S., \& Piliavin, J. A. (2004). Values: Reviving a dormant concept. Annual Review of Sociology, 359-393. https://doi.org/https://doi.org/ 10.1146/annurev.soc.30.012703.110640

Hofstede, G. (1980). Culture's consequences: International differences in work-related values. Sage.

Inbar, Y. (2016). Association between contextual dependence and replicability in psychology may be spurious. Proceedings of the $\mathrm{Na}$ tional Academy of Sciences, 113(34), E4933E4934. https : / / doi . org / 10 . 1073/pnas . 1608676113

Inglehart, R. (2015). The silent revolution: Changing values and political styles among Western publics. Princeton University Press.

John, L. K., Loewenstein, G., \& Prelec, D. (2012). Measuring the prevalence of questionable research practices with incentives for truth telling. Psychological Science, 23(5), 524532. https : / / doi . org / 10 . 1177/ 0956797611430953

Jost, J. T., \& Kruglanski, A. W. (2002). The estrangement of social constructionism and experimental social psychology: History of the rift and prospects for reconciliation. Personality and Social Psychology Review, 6(3), 168-187. https : / / doi . org / 10 . 1207/ S15327957PSPR0603_1

Kluckhohn, C. (1951). Values and value-orientations in the theory of action: An exploration in definition and classification. In T. Parsons \& E. A. Shils (Eds.), Toward a general theory of action. Harvard University Press. https :// doi . org / http : / dx . doi . org / 10 . 4159/ harvard . 9780674863507.c8

Knafo, A., \& Spinath, F. M. (2011). Genetic and environmental influences on girls' and boys' gender-typed and gender-neutral values. $\mathrm{De}$ velopmental Psychology, 47(3), 726-731. https://doi.org/10.1037/a0021910

Maio, G. R. (2010). Mental representations of social values. Advances in experimental social psychology, 42, 1-43.

Mischel, W., \& Shoda, Y. (1995). A CognitiveAffective System Theory of Personality: Reconceptualizing Situations, Dispositions, Dynamics, and Invariance in Personality Structure. Psychological Review, 102(2), 246-268. https :// doi .org/10 . 1037/0033 295X.102.2.246

Montoya, R. M., Horton, R. S., \& Kirchner, J. (2008). Is actual similarity necessary for attraction? A meta-analysis of actual and perceived sim- 
ilarity. Journal of Social and Personal Relationships, 25(6), 889-922. https://doi.org/10. 1177/0265407508096700

Moscovici, S. (1961). La psychanalyse, son image, son public [Psychoanalysis: Its image and its public]. Paris, Presses Universitaires de France. https://doi.org/10.2307/3319763

Moscovici, S. (1984). The phenomena of social representations. In R. M. Farr \& S. Moscovici (Eds.), Social representations (pp. 18-77). Cambridge, UK, Cambridge University Press.

Open Science Collaboration. (2015). Estimating the reproducibility of psychological science. Science, 349(6251), aac4716. https://doi.org/10. $1126 /$ science.aac4716

Osgood, C. E., Suci, G. J., \& Tannenbaum, P. H. (1957). The measurement of meaning. Urbana, IL, University of Illinois Press.

Pettigrew, T. F. (1998). Intergroup Contact Theory. Annual Review of Psychology, 49(1), 65-85. https://doi.org/10.1146/annurev.psych.49.1. 65

Piurko, Y., Schwartz, S. H., \& Davidov, E. (2011). Basic Personal Values and the Meaning of LeftRight Political Orientations in 20 Countries. Political Psychology, 32(4), 537-561. https: //doi.org/10.1111/j.1467-9221.2011.00828.x

Ponizovskiy, V. (2020). On Sanders, Trump, and Rhinoceroses: Quantifying subjective construals helps predict political attitudes.

Ponizovskiy, V., Grigoryan, L., Kühnen, U., \& Boehnke, K. (2019). Social construction of the value-behavior relation. Frontiers in Psychology, 10. https://doi.org/10.3389/fpsyg. 2019.00934

Renger, D., Eschert, S., Teichgräber, M. L., \& Renger, S. (2020). Internalized equality and protest against injustice: The role of disadvantaged group members' self-respect in collective action tendencies. European Journal of Social Psychology, 50(3), 547-560. https://doi.org/ 10.1002/ejsp.2637

Roccas, S., \& Schwartz, S. H. (1997). Church-state relations and the association of religiosity with values: A study of Catholics in six countries. Cross-Cultural Research, 31(4), 356-375.

Roget, P. M. (1878). Thesaurus of English words and phrases. New York, NY, Sheldon; Company. https://doi.org/10.1017/cbo9781107448735

Rohan, M. J. (2000). A rose by any name? The values construct. Personality and social psychology review, 4(3), 255-277.

Rokeach, M. (1973). The nature of human values (Vol. 438). New York, Free Press.

Rosenberg, M. J. (1956). Cognitive structure and attitudinal affect. Journal of Abnormal and So- cial Psychology, 53(3), 367-372. https://doi. org/10.1037/h0044579

Ross, L., Greene, D., \& House, P. (1977). The "false consensus effect": An egocentric bias in social perception and attribution processes. Journal of Experimental Social Psychology, 13(3), 279-301. https :// doi . org/10 .1016/ 0022-1031(77)90049-X

Ross, L., \& Ward, A. (1996). Naive realism in everyday life: Implications for social conflict and misunderstanding. In E. S. Reed, E. Turiel, \& T. Brown (Eds.), The jean piaget symposium series. values and knowledge (pp. 103-135). Lawrence Erlbaum Associates, Inc.

Sassenberg, K., \& Ditrich, L. (2019). Research in social psychology changed between 2011 and 2016: Larger sample sizes, more self-report measures, and more online studies. Advances in Methods and Practices in Psychological Science, 2(2), 107-114. https://doi.org/10. $1177 / 2515245919838781$

Savelkoul, M., Scheepers, P., Tolsma, J., \& Hagendoorn, L. (2011). Anti-Muslim attitudes in the Netherlands: Tests of contradictory hypotheses derived from ethnic competition theory and intergroup contact theory. European Sociological Review, 27(6), 741-758. https:// doi.org/10.1093/esr/jcq035

Schwartz, S. H. (1992). Universals in the content and structure of values: Theoretical advances and empirical tests in 20 countries. Advances in experimental social psychology, 25(1), 1-65.

Schwartz, S. H. (1994a). Are There Universal Aspects in the Structure and Contents of Human Values? Journal of Social Issues, 50(4), 19-45. https://doi.org/10.1111/j.1540-4560.1994. tb01196.x

Schwartz, S. H. (1994b). Beyond individualism/collectivism: New cultural dimensions of values. In U. Kim, H. C. Triandis, Ç. Kâğitçibaşi, S.-C. Choi, \& G. Yoon (Eds.), Cross-cultural research and methodology series, vol. 18. individualism and collectivism: Theory, method, and applications (pp. 85-119). Sage Publications, Inc.

Schwartz, S. H. (1996). Value priorities and behavior: Applying a theory of integrated value systems. In C. Seligman, J. Olson, \& M. Zanna (Eds.), The psychology of values: The ontario symposium, vol. 8 (pp. 1-24). Hillsdale, NJ, Erlbaum.

Schwartz, S. H. (2012). Values and religion in adolescent development: Cross-national and comparative evidence. Values, Religion, and Culture in Adolescent Development, 97122. https : / / doi . org / 10 . 1017/ CBO9781139013659.007 
Schwartz, S. H., \& Bilsky, W. (1990). Toward a theory of the universal content and structure of values: Extensions and cross-cultural replications. Journal of personality and social psychology, 58(5), 878.

Schwitzgebel, E. (2002). A phenomenal, dispositional account of belief. Nous, 36(2), 249-275. https://doi.org/10.1111/1468-0068.00370

Speaks, J. (2019). Theories of meaning. In E. N. Zalta (Ed.), Stanford encyclopedia of philosophy. Stanford, CA, Stanford University. https:// plato.stanford.edu/entries/meaning/

Stephan, W. G., \& Stephan, C. W. (2000). An integrated threat theory of prejudice. In S. Oskamp (Ed.), Reducing prejudice and discrimination (pp. 23-45). Erlbaum.

Swiatkowski, W., \& Dompnier, B. (2017). Replicability crisis in social psychology: Looking at the past to find new pathways for the future. International Review of Social Psychology, 30(1), 111-124. https://doi .org/10.5334/ irsp.66

Touhey, J. C. (1981). Replication Failures in Personality and Social Psychology. Personality and Social Psychology Bulletin, 7(4), 593-595. https://doi.org/10.1177/014616728174012

Uzefovsky, F., Döring, A. K., \& Knafo-Noam, A. (2016). Values in Middle Childhood: Social and Genetic Contributions. Social Development, 25(3), 482-502. https:// doi . org/10. $1111 /$ sode. 12155

Van Bavel, J. J., Mende-Siedlecki, P., Brady, W. J., \& Reinero, D. A. (2016a). Contextual sensitivity in scientific reproducibility. Proceedings of the National Academy of Sciences of the United States of America, 113(23), 64546459. https : / / doi . org / 10 . 1073 / pnas . 1521897113

Van Bavel, J. J., Mende-Siedlecki, P., Brady, W. J., \& Reinero, D. A. (2016b). Reply to Inbar: Contextual sensitivity helps explain the reproducibility gap between social and cognitive psychology. https://doi.org/10.1073/pnas. 1609700113

van de Vijver, F., \& Tanzer, N. K. (2004). Bias and equivalence in cross-cultural assessment: An overview. Revue europeenne de psychologie appliquee, 54(2), 119-135. https://doi.org/ 10.1016/j.erap.2003.12.004

Wigfield, A., \& Eccles, J. S. (2000). Expectancy-value theory of achievement motivation. Contemporary Educational Psychology, 25(1), 6881. https://doi.org/10.1006/ceps.1999.1015

Wright, S. C., Aron, A., McLaughlin-Volpe, T., \& Ropp, S. A. (1997). The Extended Contact Effect: Knowledge of Cross-Group Friendships and Prejudice. Journal of Personality and Social Psychology, 73(1), 73-90. https : //doi.org/10.1037/0022-3514.73.1.73

Wyer, R. S., \& Albarracin, D. (2005). Belief formation, organization, and change: Cognitive and motivational influences. In D. Albarracin, B. T. Johnson, \& M. P. Zanna (Eds.), The handbook of attitudes (pp. 273-322). New York, NY, Psychology Press. https://www.researchgate. net/publication/284330915

Yarkoni, T. (2019). The generalizability crisis. PsyArXiv, 1-26. https://doi.org/10.31234/ osf.io/jqw35 
Table 1

Definitions and examples of basic human values.

Definition

Security: Safety, harmony, and stability of society, National security, social order, clean.

of relationships, and of self.

Conformity: Restraint of actions, inclinations, and impulses likely to upset or harm others and violate social expectations or norms.

Tradition: Respect, commitment, and acceptance of the customs and ideas that traditional culture or religion provide.

Benevolence: Preservation and enhancement of the welfare of people with whom one is in frequent personal contact.

Universalism: Understanding, appreciation, tolerance, and protection for the welfare of all people and for nature.

Self-direction: Independent thought and action-choosing, creating, exploring.

Stimulation: Excitement, novelty, and challenge in life.

Hedonism: Pleasure and sensuous gratification for oneself.

Achievement: Personal success through demonstrating competence according to social standards.

Politeness, obedient, honoring parents and elders.

Humble, devout, accepting my portion in life.

Helpful, honest, forgiving.

Broad-minded, social justice, equality, protecting the environment.

Creativity, curious, freedom.

Daring, varied life, exciting life.

Pleasure, enjoying life.

Successful, capable, ambitious.

Power: Social status and prestige, control or dom- Social power, authority, wealth. inance over people and resources.

Note. Adapted with permission from Schwartz, 1994. (C) John Wiley and Sons. 